Homology, Homotopy and Applications, vol.7(1), 2005, pp.1-8

\title{
DESCENT IN CATEGORIES OF (CO)ALGEBRAS
}

\author{
BACHUKI MESABLISHVILI
}

(communicated by George Janelidze)

Abstract

The paper is devoted to the investigation of effective descent morphisms in categories of (co)algebras.

\section{Introduction}

Given a category $\mathcal{A}$ and an object $a \in \mathcal{A}$, one has the slice category $\mathcal{A} / a$, an object of which is a morphism $f: x \rightarrow a$ in $\mathcal{A}$, and a morphism $f \rightarrow f^{\prime}$ in which is a morphism $h: x \rightarrow x^{\prime}$ in $\mathcal{A}$ with $f^{\prime} h=f$. Composition and identity morphisms are as in $\mathcal{A}$.

An arbitrary morphism $p: a^{\prime} \rightarrow a$ in $\mathcal{A}$ induces a functor $p_{!}: \mathcal{A} / a^{\prime} \rightarrow \mathcal{A} / a$ sending $f: x \rightarrow a^{\prime}$ to $p f: x \rightarrow a$; and when $\mathcal{A}$ has pullbacks, this functor has the right adjoint $p^{*}: \mathcal{A} / a \rightarrow \mathcal{A} / a^{\prime}$ (known as the change-of-base functor) given by pulling back along $p$. If, in addition, $p^{*}$ is monadic, then one says that the morphism $p: a^{\prime} \rightarrow a$ is an effective $\mathcal{A}$-descent morphism.

In the present paper, we study conditions under which a morphism in the category of (co)algebras with respect to a given endofunctor is effective for descent.

We refer to M. Barr and C. Wells $[\mathbf{1}]$ and F. Borceux $[\mathbf{3}]$ for terminology and general results on monads, and to G. Janelidze and W. Tholen [5], [6] for Grothendieck descent theory; we give, however, full details of all auxiliary results that are not mentioned there explicitly.

\section{Preliminaries on Slice Categories}

In this section, we collect some basic facts on slice categories. We begin by recalling that, for any object $a$ of a category $\mathcal{A}$, the underlying object functor $\mathcal{A} / a \rightarrow \mathcal{A}$ is conservative and preserves and reflects any colimit that exists in $\mathcal{A}$. Moreover, if $\mathcal{A}$ is (finitely) complete, then $\mathcal{A} / a$ is (finitely) complete as well.

Let $U: \mathcal{A} \rightarrow \mathcal{X}$ be a functor. Since, for any object $a \in \mathcal{A}$, the functor

$$
\begin{gathered}
U_{a}: \mathcal{A} / a \rightarrow \mathcal{X} / U(a) \\
(f: x \rightarrow a) \longrightarrow(U(f): U(x) \rightarrow U(a)),
\end{gathered}
$$

Received June 29, 2004, revised December 22, 2004; published on February 4, 2005

2000 Mathematics Subject Classification: 18A20, 18A30, 18C15

Key words and phrases: Slice category, effective descent morphism, (co)algebra for an endofunctor (C) 2005, Bachuki Mesablishvili. Permission to copy for private use granted. 
makes the diagram

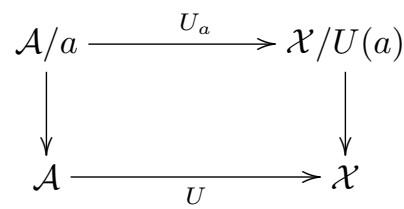

(where the vertical arrows are the forgetful functors) commute, it follows immediately from the above that:

Proposition 1. Let $U: \mathcal{A} \rightarrow \mathcal{X}$ be a functor. If $U$ is conservative, then so is the functor $U_{a}: \mathcal{A} / a \rightarrow \mathcal{X} / U(a)$. Moreover, given a small category $J$, if $\mathcal{A}$ admits and $U$ preserves $J$-colimits, then $\mathcal{A} /$ a has and $U_{a}$ preserves $J$-colimits. In particular, if $\mathcal{A}$ has and $U$ preserves coequalizers of $U$-split pairs, then the category $\mathcal{A} /$ a has and $U_{a}: \mathcal{A} / a \rightarrow \mathcal{X} / U(a)$ preserves coequalizers of $U_{a}$-split pairs.

Moreover, it is straightforward to check that:

Proposition 2. Suppose that both $\mathcal{A}$ and $\mathcal{X}$ have pullbacks and that $U$ preserves them. Then, for any morphism $p: a^{\prime} \rightarrow a$ in $\mathcal{A}$, the diagram

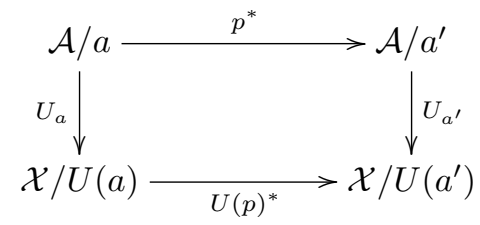

commutes up to isomorphism. Moreover, if the morphism $U(p)$ is a split epimorphism, then the natural transformation

$$
U_{a} \cdot \epsilon: U_{a} \circ p_{!} \circ p^{*} \rightarrow U_{a},
$$

where $\epsilon: p_{!} \circ p^{*} \rightarrow 1$ is the counit of the adjunction $p_{!} \dashv p^{*}$, is a split epimorphism.

Recall that a morphism is an extremal epimorphism when it does not factor through any proper subobject of its codomain.

Proposition 3. Let $U: \mathcal{A} \rightarrow \mathcal{X}$ be a conservative functor preserving monomorphisms. If $p: a^{\prime} \rightarrow a$ is a morphism in $\mathcal{A}$ such that the morphism $U(p): U\left(a^{\prime}\right) \rightarrow$ $U(a)$ in $\mathcal{X}$ is an extremal epimorphism, then $p$ is an extremal epimorphism as well. In other words, $U$ reflects extremal epimorphisms.

Proof. If $p: a^{\prime} \rightarrow a$ factorizes through a monomorphism $i: b \rightarrow a$, then, since $U$ preserves monomorphisms by assumption, $U(p)$ factorizes through the monomorphism $U(i)$; hence ( $\mathrm{U}(\mathrm{p})$ being an extremal epimorphism) $U(i)$ is an isomorphism in $\mathcal{X}$, whence $i$ is an isomorphism as well because $U$ is conservative by hypothesis.

Corollary 4. Let $\mathcal{A}$ and $\mathcal{X}$ be categories with pullbacks, and let $U: \mathcal{A} \rightarrow \mathcal{X}$ be a conservative functor that preserves pullbacks. If $p: a^{\prime} \rightarrow a$ is a morphism in $\mathcal{A}$ such that the morphism $U(p): U\left(a^{\prime}\right) \rightarrow U(a)$ in $\mathcal{X}$ is a stably-extremal epimorphism (and so in particular if $U(p)$ is a split epimorphism), then $p$ is a stably-extremal epimorphism as well. 
Proof. First observe that, since any pullback-preserving functor in particular preserves monomorphisms, it follows from the above proposition that $U$ reflects extremal epimorphisms.

Next, since $U$ preserves pullbacks by hypothesis, the image under $U$ of the pullback of $p$ along an arbitrary morphism is (isomorphic to) the pullback of $U(p)$, which is an extremal epimorphism by our assumption on $p$. But, as we just observed, the functor $U$ reflects extremal epimorphisms; so that the pullback of $p$ is an extremal epimorphism. Hence $p$ is a stably-extremal epimorphism.

Since, for any morphism $p: a^{\prime} \rightarrow a$, the functor $p^{*}: \mathcal{A} / a \rightarrow \mathcal{A} / a^{\prime}$ is conservative if and only if the morphism $p$ is an stably-extremal epimorphism (see, for instance, [4]), we have:

Proposition 5. In the situation of Corollary 2.4, the change-of-base functor $p^{*}$ : $\mathcal{A} / a \rightarrow \mathcal{A} / a^{\prime}$ is conservative.

\section{Criteria for Effective Descent}

We begin with

Theorem 6. Let $V: \mathcal{A} \rightarrow \mathcal{B}$ be a conservative functor with a left adjoint $G: \mathcal{B} \rightarrow$ $\mathcal{A}$. Suppose that there exists a commutative (up to isomorphism) diagram

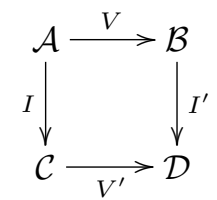

such that

(i) $\mathcal{A}$ has coequalizers of I-split pairs and I preserves them;

(ii) $\mathcal{C}$ is Cauchy complete (or, equivalently, idempotents split in $\mathcal{C}$; that is, every idempotent endomorphism $e$ in $\mathcal{C}$ has a factorization $e=i$ ir where $r i=1$ );

(iii) I' is conservative;

(iv) the natural transformation

$$
\text { I } \epsilon: I G V \rightarrow I,
$$

where $\epsilon: G V \rightarrow 1$ is the counit of the adjunction $G \dashv V$, is a split epimorphism.

Then the functor $V$ is monadic.

Proof. Suppose that $a \underset{g}{\stackrel{f}{\rightleftarrows}} a^{\prime}$ is a $V$-split pair of morphisms in $\mathcal{A}$. Then the morphisms $V(f)$ and $V(g)$ have a split coequalizer in $\mathcal{B}$; so that the pair $(V(f), V(g))$ is contractible (see [1] ). Since the natural transformation $I \epsilon: I G V \rightarrow I$ is a split epimorphism, the pair $(I(f), I(g))$ of morphisms in $\mathcal{C}$ is also contractible by Corollary 1.3 of [7]. Then, since idempotents split in $\mathcal{C}$ by hypothesis, $I(f)$ and $I(g)$ have a split coequalizer (see, for instance, $[\mathbf{2}]$ ); hence applying our assumption (i), we get 
that $f$ and $g$ have a coequalizer and this coequalizer is preserved by $I$. Moreover, the same argument as in the proof of Theorem 2.3 of [7] shows that this coequalizer is also preserved by $V$.

So, we know that

- $V$ is conservative and has the left adjoint $G$;

- $\mathcal{A}$ has and $V$ preserves coequalizers of $V$-split pairs.

Applying Beck's theorem (in the form given by Barr and Wells as Theorem 10 in [7]) now gives the monadicity of $V$.

Note that when $\mathcal{A}$ and $\mathcal{B}$ are categories with coequalizers, one may drop the condition (ii) and then our theorem is exactly the same as Theorem 2.3 in [7].

With the aid of the above theorem, we can now prove:

Theorem 7. Let $\mathcal{A}$ and $\mathcal{X}$ be categories with pullbacks, and let $U: \mathcal{A} \rightarrow \mathcal{X}$ be a conservative functor that preserves pullbacks. Suppose furthermore that

- $\mathcal{A}$ has and $U$ preserves coequalizers of $U$-split pairs;

- $\mathcal{X}$ has coequalizers.

Then, if the image under $U$ of a morphism $p: a^{\prime} \rightarrow a$ in $\mathcal{A}$ is a split epimorphism, then $p$ is an effective $\mathcal{A}$-descent morphism.

Remark 8. Observe that, under the given assumption on $U(p): U\left(a^{\prime}\right) \rightarrow U(a)$, it follows from Theorem 2.2 of [7] that the morphism $U(p)$ is an effective $\mathcal{X}$-descent morphism.

Proof. Let us first observe that, for any object $a \in \mathcal{A}$, the diagram (1) commutes (up to isomorphism), since $\mathcal{A}$ and $\mathcal{X}$ have pullbacks and $U$ preserves them by assumption. We also have that

- $p^{*}$ has the left adjoint $p_{\text {! }}$;

- $p^{*}$ is conservative (see Proposition 2.5);

- $\mathcal{X} / U(a)$ is Cauchy complete, since any category admitting coequalizers is Cauchy complete (and $\mathcal{X} / U(a)$ admits coequalizers, since so does $\mathcal{X}$ by assumption).

- since $\mathcal{A}$ has and $U$ preserves coequalizers of $U$-split pairs by hypothesis, Proposition 2.1 tells us that $\mathcal{A} / a$ has and $U_{a}: \mathcal{A} / a \rightarrow \mathcal{X} / U(a)$ preserves coequalizers of $U_{a}$-split pairs;

- the functor $U_{a^{\prime}}: \mathcal{A} / a^{\prime} \rightarrow \mathcal{X} / U\left(a^{\prime}\right)$ is conservative by Proposition 2.1;

- the natural transformation

$$
U_{a} \cdot \epsilon: U_{a} \circ p_{!} \circ p^{*} \rightarrow U_{a},
$$

where $\epsilon: p_{!} \circ p^{*} \rightarrow 1$ is the counit of the adjunction $p_{!} \dashv p^{*}$, is a split epimorphism by Proposition 2.2.

The desired result now follows from Theorem 3.1 applied to the commutative diagram (1). 
The next result gives another criterion for a functor to be monadic.

Theorem 9 ([8]). Let $\mathcal{A}$ and $\mathcal{B}$ be categories with coequalizers. A conservative functor $V: \mathcal{A} \rightarrow \mathcal{B}$ with a left adjoint is monadic if and only if there exists a commutative (up to isomorphism) diagram

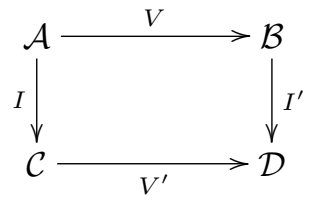

such that

(i) I preserves coequalizers of $V$-split pairs;

(ii) $I^{\prime}$ is conservative;

(iii) $V^{\prime}$ preserves coequalizers of $V^{\prime}$-split pairs.

Based on this result, we are now able to prove:

Theorem 10. Let $\mathcal{A}, \mathcal{X}$ be categories with pullbacks and coequalizers, and let $U$ : $\mathcal{A} \rightarrow \mathcal{X}$ be a conservative functor that preserves pullbacks and coequalizers. Then $U$ reflects effective descent morphisms.

Proof. We have to show that any morphism, whose image under $U$ is an effective $\mathcal{X}$-descent morphism, is an effective $\mathcal{A}$-descent morphism. Suppose therefore that $p: a^{\prime} \rightarrow a$ is morphism in $\mathcal{A}$ such that the morphism $U(p): U\left(a^{\prime}\right) \rightarrow U(a)$ is an effective $\mathcal{X}$-descent morphism.

Note that, for any object $a \in \mathcal{A}$, both $\mathcal{A} / a$ and $\mathcal{X} / U(a)$ admit pullbacks and coequalizers because $\mathcal{A}$ and $\mathcal{X}$ do so by assumption. Note also that, as in the proof of Theorem 3.2, Proposition 2.2 yields the commutative diagram (1).

Next, we have:

- $p^{*}$ has a left adjoint, namely the functor $p_{!}$;

- since $U$ preserves all coequalizers by assumption, so does the functor $U_{a}$ : $\mathcal{A} / a \rightarrow \mathcal{X} / U(a)$ (see Proposition 2.1.);

- $U_{a^{\prime}}$ is conservative by Proposition 2.1;

- by hypothesis, $U(p)^{*}$ is monadic, and hence in particular it preserves coequalizers of $U(p)^{*}$-split pairs.

According Theorem 3.4, it remains to show that the functor $p^{*}: \mathcal{A} / a \rightarrow \mathcal{A} / a^{\prime}$ is conservative. But the functor $U(p)^{*}$, being monadic by assumption, is conservative, while $U_{a}$ is conservative, since (see Proposition 2.1) $U$ is so by hypothesis. But conservativeness is a composite property (i.e. if the class of conservative functors contains $U_{2} \circ U_{1}$, then it contains $\left.U_{1}\right)$, so that the composite $U(p)^{*} \circ U_{a}$, and hence also the composite $U_{a^{\prime}} \circ p^{*}$, are conservative; therefore $p^{*}$ is conservative. This completes the proof. 
A corollary is immediate:

Corollary 11. Suppose, in addition of the hypothesis of Theorem 3.5, that the category $\mathcal{X}$ satisfies the axiom of choice (i.e., each regular epimorphism in $\mathcal{X}$ splits), then a morphism in $\mathcal{A}$ is an effective $\mathcal{A}$-descent morphism if and only if its image under $U$ is an effective $\mathcal{X}$-descent morphism.

\section{Effective Descent Morphisms in Categories of (Co)algebras}

In this section we apply the results of the previous section to obtain criteria for morphisms in a category of (co)algebras to be effective descent. We begin by recalling the definitions of algebra and coalgebra for an endofunctor.

Let $\mathcal{X}$ be a category, and let $\Gamma: \mathcal{X} \rightarrow \mathcal{X}$ be an endofunctor. A $\Gamma$-algebra is a pair $(x, \alpha)$, where $x$ is an object in $\mathcal{X}$ and $\alpha: \Gamma(x) \rightarrow x$ is a morphism in $\mathcal{X}$. Given two $\Gamma$-algebras $(x, \alpha)$ and $\left(x^{\prime}, \alpha^{\prime}\right)$, a $\Gamma$-morphism $p:\left(x^{\prime}, \alpha^{\prime}\right) \rightarrow(x, \alpha)$ is a morphism $p: x^{\prime} \rightarrow x$ in $\mathcal{X}$ for which

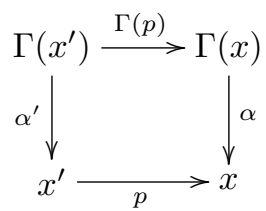

commutes. The $\Gamma$-algebras and their morphisms form a category, denoted $\mathcal{X}^{\Gamma}$. Dually, one has the category of $\Gamma$-coalgebras and their morphisms, denoted $\mathcal{X}_{\Gamma}$.

For a given endofunctor $\Gamma: \mathcal{X} \rightarrow \mathcal{X}$, the categories $\mathcal{X}^{\Gamma}$ and $\mathcal{X}_{\Gamma}$ are equipped with the evident forgetful functors

$$
U^{\Gamma}: \mathcal{X}^{\Gamma} \rightarrow \mathcal{X}
$$

and

$$
U_{\Gamma}: \mathcal{X}_{\Gamma} \rightarrow \mathcal{X}
$$

respectively.

The following results are mentioned for example in [1]:

Proposition 12. Let $\Gamma: \mathcal{X} \rightarrow \mathcal{X}$ be an endofunctor on a category $\mathcal{X}$. Then

(i) the forgetful functor $U^{\Gamma}$ is conservative;

(ii) the category $\mathcal{X}^{\Gamma}$ has and the functor $U^{\Gamma}$ preserves coequalizers of $U^{\Gamma}$-split pairs;

(iii) the functor $U^{\Gamma}$ creates (and hence preserves) whatever limits that exist in $\mathcal{X}$.

Note that the functor $U^{\Gamma}$ is obviously monadic if it has a left adjoint.

Just as in the case of algebras for monads (see, for example, $[\mathbf{3}]$ ), one can prove that: 
Proposition 13. Let $\Gamma: \mathcal{X} \rightarrow \mathcal{X}$ be an endofunctor on a category $\mathcal{X}$. Then the forgetful functor $U^{\Gamma}: \mathcal{X}^{\Gamma} \rightarrow \mathcal{X}$ creates (and hence preserves) any types of limits which exist in $\mathcal{X}$ and are preserved by $\Gamma$.

We shall assume from now on that our category $\mathcal{X}$ admits pullbacks and coequalizers.

Applying Theorem 3.2 and Proposition 4.1, we obtain:

Theorem 14. Let $\Gamma: \mathcal{X} \rightarrow \mathcal{X}$ be an endofunctor on $\mathcal{X}$. Then any morphism of the category $\mathcal{X}^{\Gamma}$ whose image under the functor $U^{\Gamma}$ is a split epimorphism is an effective $\mathcal{X}^{\Gamma}$-descent morphism.

Consider now the case where $\Gamma$ preserves pullbacks.

Theorem 15. If $\Gamma: \mathcal{X} \rightarrow \mathcal{X}$ preserve pullbacks, then the functor $U_{\Gamma}: \mathcal{X}_{\Gamma} \rightarrow \mathcal{X}$ reflects effective descent morphisms.

Proof. We observe that

- $U_{\Gamma}: \mathcal{X}_{\Gamma} \rightarrow \mathcal{X}$ is conservative (by the dual of Proposition 4.1 (i));

- $\mathcal{X}_{\Gamma}$ has and $U_{\Gamma}: \mathcal{X}_{\Gamma} \rightarrow \mathcal{X}$ preserves all coequalizers (by the dual of Proposition 4.1 (iii));

- $\mathcal{X}_{\Gamma}$ has and $U_{\Gamma}: \mathcal{X}_{\Gamma} \rightarrow \mathcal{X}$ preserves pullbacks (by the dual of Proposition 4.2, since $\mathcal{X}$ has and $\Gamma$ preserves pullbacks by assumption).

This means that, for the functor $U_{\Gamma}: \mathcal{X}_{\Gamma} \rightarrow \mathcal{X}$, we have verified all the hypothesis of Theorem 3.5. Hence any morphisms in $\mathcal{X}_{\Gamma}$ whose image under the functor $U_{\Gamma}: \mathcal{X}_{\Gamma} \rightarrow$ $\mathcal{X}$ is an effective $\mathcal{X}$-descent morphism, is an effective descent $\mathcal{X}_{\Gamma}$-morphism.

Suppose now that $\Gamma: \mathcal{X} \rightarrow \mathcal{X}$ preserves reflexive coequalizers. Then, by Proposition 4.2, the category $\mathcal{X}^{\Gamma}$ has and the functor $U^{\Gamma}: \mathcal{X}^{\Gamma} \rightarrow \mathcal{X}$ preserves all reflexive coequalizers. We now put together Proposition 4.1 and Theorem 3.5 to obtain the following

Theorem 16. Suppose that $\Gamma: \mathcal{X} \rightarrow \mathcal{X}$ preserve reflexive coequalizers. Then the functor $U^{\Gamma}: \mathcal{X}^{\Gamma} \rightarrow \mathcal{X}$ reflects effective descent morphisms.

Note that when $\mathcal{X}$ is a (Barr) exact category, the characterization of effective descent morphisms of algebras is obtained in [9].

Finally we note that the results of this section remain true if one replaces $\Gamma$ by the functor-part of a (co)monad on $\mathcal{X}$ and the category of $\Gamma$-(co)algebras by the category of (co)algebras with respect to the given (co)monad.

\section{References}

[1] M. Barr and C. Wells, Toposes, Triples, and Theories, Grundlehren der Math. Wissenschaften 278, Springer-Verlag, 1985.

[2] M. Barr, The Point of Empty Set, Cahiers Topologie Géom. Différentielle Catégoriques 13 (1972), 357-368. 
[3] F. Borceux, Handbook of Categorical Algebra, vol. 2, Cambridge University Press, 1994.

[4] G. Janelidze and W. Tholen, How Algebraic is the Change-of-Base Functor?, Lecture Notes in Mathematics 1488, Springer, Berlin (1991), 174-186.

[5] G. Janelidze and W. Tholen, Facets of Descent, I, Appl. Categorical Structures 2 (1994), 245-281.

[6] G. Janelidze and W. Tholen, Facets of Descent, II, Appl. Categorical Structures 5 (1997), 229-248.

[7] G. Janelidze and W. Tholen, Facets of Descent, III : Monadic Descent for Rings and Algebras, Appl. Categorical Structures 12, 5-6 (2004), 461-477.

[8] B. Mesablishvili, Descent Thoery for Schemes, Appl. Categorical Structures 12, 5-6 (2004), 485-512 .

[9] A. H. Roque, Effective Descent Morphisms in Some Quasivarieties of Algebraic, Relational, and More General Structures, Appl. Categorical Structures 12, 5-6 (2004), 513-525.

This article may be accessed via WWW at http://www.rmi.acnet.ge/hha/ or by anonymous ftp at

ftp://ftp.rmi.acnet.ge/pub/hha/volumes/2005/n1a1/v7n1a1.(dvi,ps,pdf)

Bachuki Mesablishvili bachi@rmi.acnet.ge

A. Razmadze Mathematical Institute

Georgian Academy of Sciences

M. Aleksidze st., 1

0193 Tbilisi,

Georgia 